ESTUDIOS / RESEARCH STUDIES

\title{
Calidad y características de los sitios web de los hospitales españoles de gran tamaño
}

\author{
Manuel-Ángel Calvo-Calvo \\ Departamento de Enfermería; Facultad de Enfermería, Fisioterapia y Podología, Universidad de Sevilla. \\ Hospital Universitario Virgen del Rocío. Sevilla. \\ Correo-e: macalvo@us.es
}

Recibido: 14-12-2012; 2a version: 23-04-2013; 3a version: 06-06-2013; Aceptado: 18-07-2013.

Cómo citar este artículo/Citation: Calvo-Calvo, M. A. (2014). Calidad y características de los sitios web de los hospitales españoles de gran tamaño. Revista Española de Documentación Científica, 37(1):e032. doi: http://dx.doi.org/10.3989/ redc. 2014.1.1049

Resumen: El objetivo es evaluar la calidad y describir las características de los sitios web de los hospitales españoles con más de 500 camas. Estudio descriptivo, observacional, de corte transversal, que evalúa la calidad de 53 sitios web de hospitales españoles con más de 500 camas. Se analizan la accesibilidad, usabilidad, interactividad, actualización de la información, referentes de calidad y contenidos presentados en los sitios web. Sólo 22 (41,5\%) de las webs estudiadas superaron la mitad de los puntos posibles. Por tanto, como media, la calidad de las webs de los grandes hospitales españoles analizados es baja. Las webs evaluadas destacan por su accesibilidad y usabilidad, y por tener secciones específicas para profesionales y proveedores. Igualmente, las webs de los hospitales estudiados se caracterizan por una escasa incorporación de herramientas para interactuar con los usuarios, por presentar una deficiente información sobre salud, enfermedades, listas de espera y actividades asistenciales, así como por una deficitaria actualización de sus contenidos y la casi nula adhesión a referentes de calidad. En conclusión, los sitios webs de los hospitales españoles de gran tamaño, además de ser espacios informativos, deberían facilitar más interacción, participación y colaboración de los usuarios de los hospitales.

Palabras clave: evaluación de calidad; sitios web; hospitales con más de 500 camas; tecnología de la información; Internet; accesibilidad; usabilidad; interactividad; actualización de contenidos.

\section{Quality and characteristics of websites of large Spanish hospitals}

Abstract: The objective is to assess the quality and describe characteristics of websites of large Spanish hospitals, i.e. minimum 500 beds. Cross-sectional descriptive study is made, to evaluate the global quality, accessibility, usability, interactivity, updating, quality model and information given in 53 different websites belonging to Spanish hospitals with more than 500 beds. Only $22(41.5 \%)$ of the websites analyzed fall into the upper half of the scale used. As such, the mean quality of these websites is shown to be low. On one hand, both accessibility and usability of the evaluated websites were notable, with specific sections for health professionals and hospital suppliers. On the other, the websites were characterized by a limited incorporation of tools for interacting with users, a poor presentation of information about health, diseases, waiting lists and care activities, as well as a deficient updating of their content and almost no adherence to quality references. In short, the websites of large Spanish hospitals are simple informational spaces that don't facilitate the interaction, participation and collaboration of hospital users. In conclusion, websites of large Spanish hospitals, in addition to being informative spaces, should facilitate more interaction, participation and collaboration with hospital users.

Keywords: quality evaluation; websites; hospital bed capacity 500 and over; information technology; Internet; accessibility; usability; interactivity; content update.

Copyright: (c) 2014 CSIC. Este es un artículo de acceso abierto distribuido bajo los términos de la licencia Creative Commons Attribution-Non Commercial (by-nc) Spain 3.0. 


\section{INTRODUCCIÓN}

La rapidez, economía y versatilidad con que fluye la comunicación y la información a través de Internet, lo ha convertido en un medio fundamental para la transmisión del conocimiento y la interacción social (Arencibia-Jiménez y Aibar-Remón, 2007). Gracias a estas características, Internet ha conseguido que multitud de personas y organizaciones participen de esa revolución tecnológica creando sus propios sitios web o colecciones de páginas electrónicas jerarquizadas, interconectadas por enlaces y accesibles a través de redes.

Las organizaciones sanitarias no han quedado atrás y también han participado de esta revolución tecnológica, pues a través de sitios web ofrecen información y servicios de salud a ciudadanos y a profesionales sanitarios.

En los últimos años han proliferado las webs de hospitales (Arencibia-Jiménez y Aibar-Remón, 2007; Navarro-Arnedo, 2009). De este modo, estas instituciones encargadas de la asistencia sanitaria especializada y complementaria a una población determinada, se hacen presentes y visibles en la red, se comunican con sus usuarios e intentan satisfacer sus demandas, prestando un servicio público mediante herramientas de información, comunicación e interacción, y de acceso a determinados servicios de salud (Doblas, 2007).

Por esas razones, Rafe y Monfaredzadeh (2012) sostienen que el uso de Internet posibilita que los hospitales proporcionen más servicios y mejor cualificados, con una disminución de los gastos, un ahorro de tiempo e incluso permita a los hospitales superar a sus competidores. A esto, se une la ventaja que ofrecen las webs de los hospitales de posibilitar la participación de los ciudadanos en su propio proceso de salud-enfermedad (Díaz y Chaín, 2008).

No obstante, se observa que las características de calidad y contenidos en las webs de hospitales son muy variables (Conesa y Aguinaga, 2009), por lo que surge la necesidad de asegurar el rigor y calidad en los contenidos de esas webs sanitarias (Arencibia-Jiménez y Aibar-Remón, 2007). Consciente de ello, ya en el año 2000, la Unión Europea impulsó el proyecto "eEurope 2002" para desarrollar los criterios de calidad exigibles a las páginas web del ámbito de la salud, con el propósito de aumentar el uso y la confianza del usuario hacia este tipo de sitios, y fomentar una mejor práctica en el desarrollo de webs sanitarias (Domínguez-Castro e Iñesta-García, 2004; Jiménez y otros, 2009a). En diciembre de 2012, la Comisión Europea publicó un nuevo plan, el "eHealth Action Plan 2012-2020-Innovative healthcare for the 21 st century", que actualiza al proyecto anterior y que pretende seguir innovando en eSalud mediante el desarrollo de las nuevas tecnologías, para garantizar a la ciudadanía europea una atención sanitaria de mejor calidad y más segura (European Commission, 2013).
Actualmente, la medición de la calidad de las webs sanitarias sigue siendo un tema de gran relevancia, lo cual justifica que en los últimos años se hayan desarrollado múltiples instrumentos y escalas para su cuantificación (Jiménez y otros, 2009a), algunos incluso desde una perspectiva cualitativa (Rafe y Monfaredzadeh, 2012). De este modo, hay estudios que evalúan la calidad de webs dedicadas a enfermedades (Conesa y otros, 2011), de webs sanitarias en general (Conesa y otros, 2011; Conesa y Aguinaga, 2009), y de sitios web de salud para mayores (Jiménez y otros, 2009b). Otros autores han evaluado la calidad de las webs de unidades hospitalarias de cuidados intensivos (Navarro-Arnedo, 2009), y de las webs relacionadas con medicamentos (Domínguez-Castro e Iñesta-García, 2004; Jiménez y otros, 2009a). Igualmente, hay estudios de la adecuación de los códigos de conducta para información biomédica en Internet (Azpilicueta y otros, 2007), e incluso se han diseñado cuestionarios para evaluar webs sanitarias con criterios europeos (Bermúdez-Tamayo y otros, 2006).

Más específicamente, existen diversos trabajos sobre webs de hospitales, como el de ArencibiaJiménez y Aibar-Remón (2007), en el que se comparan las webs de hospitales españoles y norteamericanos. Respecto a los hospitales españoles, estos autores concluyeron que más de la tercera parte no tenían página web y que la mayoría no presentaban en sus webs información útil para el usuario, eran deficitarios en actualización, en adhesión a algún sello acreditador de calidad de la web y en usabilidad o facilidad de manejo del sitio web. Por el contrario, las webs de los hospitales españoles destacaban por ofrecer facilidades a los usuarios para contactar con el hospital y en disponer de apartados específicos para profesionales del hospital.

Mira y otros (2006) estudiaron la calidad de las webs de los hospitales públicos españoles, evaluando la accesibilidad a las webs y la legibilidad de sus contenidos mediante 73 criterios, obteniendo que la legibilidad es satisfactoria en el $64 \%$ de las webs pero que ninguno de los sitios web evaluados cumplía los requisitos necesarios para ser considerados accesibles. Díaz y Chaín (2008) analizaron las webs de los hospitales de referencia de cada provincia de España y concluyeron que la calidad de sus contenidos es insuficiente, que la mayoría de las webs no facilitan la interacción con los pacientes y tampoco presentan información suficiente, dado que en un alto porcentaje no contienen guías explicativas para pacientes, ni informan sobre servicios de interés para usuarios, como la ubicación del servicio de admisión o el de atención al paciente. Tampoco las webs estudiadas por esos autores incluían carta de derechos y deberes de los pacientes, los nombres e información de contacto con médicos por especialidades, ni los cargos directivos del hospital. 
Otro autor (Navarro-Arnedo, 2008) estudió la presencia de enfermería en las webs de los hospitales españoles, siendo muy reducida, pues únicamente el $10 \%$ de webs tenía una sección de enfermería. También concluyó que de todos los hospitales españoles que estudió, el $45 \%$ dispone de web, la mayoría ofrecen datos de localización del hospital y sus servicios, y sólo un tercio informa de su actividad asistencial, docente e investigadora.

A pesar de estos trabajos se desconoce la calidad y características de las webs de los hospitales españoles de mayor tamaño, en cuanto al número de camas, por lo que el objeto de esta investigación es dar respuesta a este aspecto desconocido.

El propósito de este trabajo, evaluar la calidad y características de los sitios webs de esos grandes hospitales españoles, tiene entidad suficiente como para constituir un objeto de estudio específico. En efecto, baste señalar que esos hospitales, por sus grandes dimensiones, suelen ser los centros de referencia para los habitantes de una zona geográfica del país, por lo que una parte importante de la población accede a sus webs demandando servicios sanitarios e información de salud.

También se justifica la pertinencia de este trabajo por la influencia que la calidad y contenidos de las webs pueden tener en el acceso de los usuarios y profesionales sanitarios, así como por los beneficios derivados de su interacción. De ahí la importancia que el análisis de las deficiencias funcionales y estructurales de las webs tiene para mejorar los servicios hospitalarios, presenciales y online, para los ciudadanos.

Igualmente, conocer las carencias de las webs evaluadas puede contribuir a fomentar una mejor práctica de los hospitales en el desarrollo de sus sitios web (Domínguez-Castro e Iñesta-García, 2004), con el consiguiente incremento del rendimiento de esas webs (Ramos y Clabo, 2008).

En consecuencia, el objetivo general de esta investigación fue evaluar la calidad y describir las características de los sitios web de los hospitales españoles de gran tamaño. Como objetivos específicos se propusieron los siguientes:

- Averiguar cuántos hospitales españoles, con más de 500 camas, poseen sitio web.

- Conocer la calidad global de los sitios webs de los hospitales españoles de gran tamaño, en función de las características técnicas presentes o ausentes en esos sitios.

- Analizar en las webs de los hospitales españoles con más de 500 camas, las características de "Accesibilidad", "Usabilidad", "Interactividad", "Información presentada", "Actualización de contenidos", "Referentes de calidad", "Información para profesionales" e "Información para proveedores" de los hospitales.

\section{METODOLOGÍA}

Se realizó un estudio observacional, descriptivo, de corte transversal en el que durante el mes de julio de 2012 se evaluó la calidad y características de las webs de los hospitales españoles de gran tamaño, aplicando sobre esta población inicial dos criterios de elegibilidad o de inclusión en el estudio. El primer criterio fue que el hospital debería ser de gran tamaño, estableciéndose que serían aquellos con 500 camas o más. El segundo criterio de inclusión fue que el hospital con más de 500 camas debería poseer sitio web, y para considerar que un hospital tenía sitio web se aplicó el criterio de Ramos y Clabo (2008). Este criterio establece que un sitio web es un conjunto de páginas electrónicas que parten de una página de inicio común, "que aparecen interrelacionadas jerárquicamente mediante enlaces internos y que conforman una unidad documental en función de sus contenidos, y también una unidad institucional diferenciada e independiente, si nos atenemos a la organización responsable del sitio web" (Ramos y Clabo, 2008).

En consecuencia, se excluyeron de esta investigación aquellos hospitales con más de 500 camas que carecían de sitio web propio. Es decir, no se tuvieron en cuenta a los grandes hospitales que no poseían un espacio específico Web, ni a aquellos cuyas páginas electrónicas estaban alojadas en el sitio web de otras entidades, pues de acuerdo con nuestro segundo criterio de elegibilidad, esas páginas electrónicas de hospitales no constituyen unidades documentales ni sitios webs independientes ni diferenciados, sino que son secciones de webs de otras entidades.

Para identificar a los hospitales españoles de gran tamaño se recurrió al Catálogo Nacional de Hospitales 2012 de España (Ministerio de Sanidad, Servicios Sociales e Igualdad, 2012), y según el primer criterio de elegibilidad, de los 790 hospitales recogidos en ese catálogo, resulta que 78 hospitales tenían 500 camas o más, por lo que esos hospitales constituyeron la población o censo inicial de estudio de esta investigación (Anexo I).

De esos 78 grandes hospitales de España, 73 $(93,6 \%)$ son hospitales generales con áreas de medicina, cirugía, obstetricia y ginecología, y 5 $(6,4 \%)$ son hospitales especializados en atender exclusivamente patologías psiquiátricas. Excepto un hospital $(1,28 \%)$, los $77(98,7 \%)$ restantes están acreditados para impartir formación a postgraduados en algunas especialidades médicas, y según su dependencia funcional, $70(89,7 \%)$ hospitales pertenecen a entidades públicas y $8(10,3 \%)$ a entidades privadas. (Ministerio de Sanidad, Servicios Sociales e Igualdad, 2012).

Posteriormente, para averiguar cuántos hospitales españoles de gran tamaño tenían sitio web se realizó una búsqueda en Internet mediante Google. En este buscador se introducía el nombre completo de cada hospital y sobre los resultados obtenidos 
se aplicó el segundo criterio de elegibilidad. Al aplicar ese criterio, resultó que de los 78 hospitales españoles con más de 500 camas, 25 (32,05\%) carecían de web y $53(67,95 \%)$ hospitales tenían sitio web (Anexo I), constituyendo estos últimos el censo definitivo de esta investigación.

De los 25 hospitales sin sitio web, en 14 (56\%) no se encontró espacio propio ni específico del hospital en Internet y de los 7 (28\%) hospitales restantes se halló una página electrónica alojada en la web del organismo responsable de cada hospital, con una breve información general del hospital. Igualmente, en $4(16 \%)$ hospitales se consideró que tampoco poseían sitio web, pues aunque tenían varias páginas electrónicas interrelacionadas, estas no constituían una unidad documental e institucional independiente ni diferenciada, ya que estaban alojadas en secciones de las webs de las organizaciones a las que pertenecían esos hospitales (Anexo I).

Para conocer la calidad y características de las 53 webs de los hospitales españoles de más de 500 camas se estudiaron ocho variables mediante un cuestionario de evaluación. Para evaluar cada variable se establecieron unos criterios y unas puntuaciones máximas (Anexo II). Las ocho variables y las puntuaciones máximas que se podían obtener se detallan en la tabla I:

- Accesibilidad: 20 puntos. Se refiere al diseño del sitio web que permita "que todas las personas, incluidas aquellas con discapacidades, puedan percibir, entender, navegar e interactuar con la web, e incluso aportar contenidos" (Jiménez y otros, 2009b).

- Usabilidad: 20 puntos. Es la facilidad del manejo y de la navegación por la página web.

- Interactividad y relación con los usuarios: 50 puntos. Se refiere a la existencia o no de relación virtual entre el hospital y usuarios a través del sitio web.

- Información presentada: 120 puntos. Se analiza si aparece información relevante y de interés para el usuario, sobre el sitio web y del hospital.

- Actualización de los contenidos: 10 puntos. Existencia en la web de fecha de edición y de actualización de contenidos.

- Referentes de calidad: 10 puntos. Es la adhesión voluntaria del sitio web a algún sello de calidad reconocido específicamente para las webs sanitarias.

- Información para el profesional: 20 puntos. Describe si existe en el sitio web una sección específica para el profesional del hospital.

- Información para proveedores: 10 puntos. Determina si en la web existe una sección específica para los proveedores del hospital.
Respecto a la accesibilidad, al igual que en los estudios de Jiménez y otros (2009b) y de Ramos y Clabo (2008), cada sitio web se evaluó mediante la herramienta automática del Test de Accesibilidad Web (TAW), desarrollada por el Centro Tecnológico de la Información y de la Comunicación (http:// www.tawdis.net). Con esta herramienta se calculó, para cada web, el número de problemas o errores de accesibilidad encontrados en los tres niveles de prioridad WAI-A, WAI-AA y WAI-AAA (Jiménez y otros, 2009b; Ramos y Clabo, 2008).

Esta variable se cuantificó de la siguiente manera: si tras el análisis de la web con la herramienta TAW, el nivel A presentaba un determinado número de problemas de accesibilidad, se multiplicaba dicho número de problemas por 0,4 y el resultado se le restaba a 12 puntos. Cuando el nivel A presentaba cero problemas de accesibilidad se evaluaba el nivel $A A$, multiplicando el número de problemas de este nivel por 0,2 y restando su resultado a 16 puntos. Si el nivel AA presentaba cero problemas, se evaluaba el nivel AAA, multiplicando el número de problemas de este nivel por 0,1 y restando su resultado a 20 puntos (Anexo II).

Para las restantes variables se tuvieron en cuenta los criterios propuestos en el cuestionario de Arencibia-Jiménez y Aibar-Remón (2007), dado que sus autores comprobaron que dicho cuestionario presenta una consistencia entre observadores y validez de contenido, adecuada y suficiente como para generalizar su empleo.

Sin embargo, sobre ese cuestionario se hicieron algunas modificaciones para actualizarlo, y así, en la variable "Usabilidad" se estableció que las webs de hospitales deberían estar optimizadas como mínimo para navegadores tipo Internet Explorer 8.0 y similares (Anexo II). También, se consideró conveniente eliminar el indicador de "poseer un icono de impresión para imprimir información en formato html", pues parece que la existencia de este icono no es una ventaja importante para el usuario con los actuales navegadores que permiten la impresión de documentos.

Para estudiar la variable "Interactividad y relación con los usuarios" se añadió el criterio de si "el hospital está presente en alguna una red social y existen enlaces a esas redes desde la web del hospital", puntuándose este criterio sobre 10 puntos (Anexo II).

Igualmente, para estudiar la variable "Información presentada" se añadieron dos nuevos criterios para evaluar "si existen secciones de prensa" y de "investigación y/o docencia", siendo valorados cada uno de esos criterios sobre 10 puntos. Además, el criterio 6 de esta variable, "Información presentada", se amplió a "identificación del personal médico y de enfermería" (Anexo II).

Para estudiar la variable "Referentes de calidad" se evaluó si la web se adhería a algún criterio de 
calidad específico para webs sanitarias, como el código Hon Code de la Health On the Net Foundation (http://www.hon.ch/HONcode/Patients/Visitor/visitor.html), el sello de la Web Médica Acreditada (Web Médica Acreditada, 2012), o el criterio de calidad para webs sanitarias de la Agencia de Calidad Sanitaria de Andalucía (Agencia de Calidad Sanitaria de Andalucía, 2012).

También se consideró pertinente añadir el estudio de una nueva variable, que no se contemplaba en el cuestionario de Arencibia-Jiménez y AibarRemón (2007), para conocer si en las webs existe información o sección para proveedores del hospital. A esta nueva variable se le asignó un valor de 10 puntos, que se obtendría en su totalidad si en la web existía una sección específica para proveedores del hospital (Anexo II).

Las variables "Actualización de los contenidos" e "Información para el profesional" se evaluaron sin modificar el cuestionario de Arencibia-Jiménez y Aibar-Remón (2007), pues los criterios propuestos por esos autores se consideraron suficientes y adecuados (Anexo II).

Según esto, la puntuación máxima posible que podría obtener cada web en la evaluación de todas las variables fue de 260 puntos, por lo que aplicando el mismo criterio que Conesa y otros (2011), se consideraría en este estudio que una web sería de calidad si su puntuación global superaba la mitad de la puntuación total posible, es decir, si superaba 130 puntos.

El autor del trabajo analizó los sitios web por lo que se evitó la subjetividad y la variación intraoperador, no teniendo que adoptar otras medidas para afrontar fuentes potenciales de sesgo. Para evaluar la calidad global de las webs se calculó el cumplimiento de los criterios de cada una de las variables (Anexo II), y luego se sumaron las puntuaciones de los criterios evaluados, tanto individualmente para cada variable como en el conjunto de todas ellas. De estas variables cuantitativas se calculó la media aritmética, la desviación están- dar, cuartiles y los valores mínimos y máximos obtenidos. Para conocer las características de las webs se sumaron los criterios que se cumplían y los que no en cada variable, y de estas variables cualitativas se calculó su frecuencia y el porcentaje. El análisis estadístico se realizó con el software IBM SPSS 21.0 para Windows.

\section{RESULTADOS}

\section{Calidad de las webs de los hospitales españo- les con más de 500 camas}

Las 53 webs de hospitales estudiadas obtuvieron una puntuación media de 126,6 (DE 23,15) sobre 260 puntos posibles, siendo 77 y 175,6 puntos, los valores mínimo y máximo hallados. Los cuartiles 1 , 2 y 3 tomaron los valores de 113,$8 ; 126,8$ y 143,9 puntos respectivamente. Sólo $22(41,5 \%)$ webs superaron la mitad de los puntos posibles, es decir, 130 puntos (Anexo III)

Respecto a las ocho variables estudiadas para medir la calidad de las webs, la "Accesibilidad", "Usabilidad", la "Información para el profesional" y la "Información para proveedores" superaron la mitad de la máxima puntuación posible que podía obtenerse para cada una de esas variables. Sin embargo, cada una de las cuatro variables restantes no alcanzaban la mitad de su máxima puntuación posible (Tabla I).

\section{Accesibilidad}

Las webs estudiadas obtuvieron en "Accesibilidad" una media de 12,37 (DE 5,29) puntos sobre 20 puntos posibles, con valores mínimos y máximos de 0 y 20 puntos respectivamente (Tabla I). De las 53 webs evaluadas, $27(51,92 \%)$ webs no cumplían ninguno de los tres criterios de nivel de accesibilidad, $14(26,93 \%)$ cumplieron sólo el nivel WAI-A, $9(17,30 \%)$ webs cumplieron los niveles WAI-A y WAI-AA simultáneamente, y $2(3,85 \%)$ cumplieron los tres niveles WAI-A, WAI-AA y WAIAAA, también simultáneamente.

Tabla I. Puntuaciones obtenidas por las webs de los hospitales españoles de gran tamaño

\begin{tabular}{|c|c|c|c|c|c|}
\hline Variables & $\begin{array}{l}\text { Valor } \\
\text { mínimo }\end{array}$ & $\begin{array}{l}\text { Valor } \\
\text { máximo }\end{array}$ & Media & DE & $\begin{array}{l}\text { Puntuación } \\
\text { máxima posible }\end{array}$ \\
\hline Accesibilidad & 0 & 20 & 12,37 & 5,29 & 20 \\
\hline Usabilidad & 6 & 17 & 12,83 & 2,95 & 20 \\
\hline Interactividad & 0 & 35 & 18,77 & 8,4 & 50 \\
\hline Información presentada & 31 & 84 & 57,42 & 12,37 & 120 \\
\hline Actualización de contenidos & 0 & 10 & 4,28 & 3,16 & 10 \\
\hline Referentes de calidad & 0 & 10 & 0,75 & 2,67 & 10 \\
\hline Información para el profesional & 0 & 20 & 13,58 & 9,43 & 20 \\
\hline Información para proveedores & 0 & 10 & 6,6 & 4,78 & 10 \\
\hline
\end{tabular}

DE: desviación estándar 


\section{Usabilidad}

Los 53 sitios web de hospitales estudiados obtuvieron en "Usabilidad" una media de 12,83 (DE $2,95)$ puntos sobre 20 puntos posibles, con valores mínimos y máximos de 6 y 17 puntos respectivamente (Tabla I).

Como se observa en la Tabla II, gran parte de las webs de hospitales estudiadas cumplen los criterios con que se ha evaluado esta variable "Usabilidad", destacando que el criterio «los enlaces describen la URL o el nombre del sitio web enlazado y se puede acceder directamente» es cumplido por la totalidad de las webs. Conviene aclarar también que de las $43(81,1 \%)$ webs que poseían herramienta de búsqueda de contenidos, $15(28,3 \%)$ eran herramientas de búsqueda avanzada, y $28(52,8 \%)$ eran herramientas de búsqueda normal (Tabla II).

\section{Interactividad y relación con el paciente}

Las webs evaluadas obtuvieron en "Interactividad" una media de $18,77(\mathrm{DE} 8,4)$ puntos sobre 50 puntos posibles, con valores mínimos y máximos de 0 y 35 puntos respectivamente (Tabla I).
Destaca que más de la mitad de las webs de hospitales estudiados facilitan un email, dirección postal, teléfono y/o fax para contactar con el Servicio de Admisión/Atención al Paciente, y en ellos existe buzón de sugerencias. Por el contrario, sólo 7 $(13,2 \%)$ webs facilitan un email para contactar con los distintos servicios del hospital y únicamente 2 $(3,8 \%)$ hospitales ofrecen la posibilidad de pedir cita médica a través de la web. Igualmente, sólo $7(13,2 \%)$ de los hospitales estudiados están presentes en alguna red o medio social, como Twitter, Facebook, YouTube, Flickr o en blogs, y disponen de enlaces a esos medios sociales desde sus webs (Tabla II).

\section{Información presentada en la web}

Las webs estudiadas obtuvieron en esta variable "Información presentada", una media de 57,42 (DE 12,37) puntos sobre 120 puntos posibles, con valores mínimos y máximos de 31 y 84 puntos respectivamente (Tabla I).

Destaca que el criterio "Guía para el paciente", que a su vez se evaluaba mediante seis subcriterios sobre un total de 12 puntos (Anexo II), obtuvo una puntuación

Tabla II. Resultados de las variables "Usabilidad" e "Interactividad" del sitio web

\begin{tabular}{|c|c|c|}
\hline \multirow{2}{*}{ Variables y criterios } & Sí lo cumple & No lo cumple \\
\hline & n $(\%)^{a}$ & n $(\%)^{a}$ \\
\hline \multicolumn{3}{|l|}{ Usabilidad } \\
\hline Tiempos de descarga del sitio web menor a 5 segundos con ADSL & $49(92,45)$ & $4(7,55)$ \\
\hline Mantiene el menú de navegación principal abierto en todas las páginas & $50(94,3)$ & $3(5,7)$ \\
\hline Optimización para navegar con Internet Explorer $8.0^{\circledR}$ y similares & $52(98,1)$ & $1(1,9)$ \\
\hline Existe herramienta de búsqueda de contenidos del sitio web & $43(81,1)$ & $10(18,9)$ \\
\hline Indican el tamaño, formato y/o tiempo de descarga de archivo & $38(71,7)$ & $15(28,3)$ \\
\hline Hay enlaces externos a otros sitios web & $34(64,2)$ & $19(35,8)$ \\
\hline $\begin{array}{l}\text { Los enlaces describen la URL o el nombre del sitio web enlazado y se puede acceder } \\
\text { directamente }\end{array}$ & $53(100)$ & $0(0)$ \\
\hline Existe mapa del sitio web & $30(56,6)$ & $23(43,4)$ \\
\hline \multicolumn{3}{|l|}{ Interactividad y relación con los usuarios } \\
\hline $\begin{array}{l}\text { Existe email para consulta/solicitud de información (Servicio de Admisión o Atención al } \\
\text { Paciente) }\end{array}$ & $28(52,8)$ & $25(47,2)$ \\
\hline $\begin{array}{l}\text { Facilitan dirección postal y teléfono y/o fax de información (Servicio de Admisión o } \\
\text { Atención al Paciente) }\end{array}$ & $46(86,8)$ & $7(13,2)$ \\
\hline Existe email para consulta/solicitud de información con los Servicios del hospital & $7(13,2)$ & $46(86,8)$ \\
\hline Facilitan dirección postal y teléfono y/o fax de contacto con Servicios del hospital & $15(28,3)$ & $28(71,7)$ \\
\hline Existe buzón de sugerencias en el sitio web & $38(71,7)$ & $15(28,3)$ \\
\hline Hay posibilidad de pedir cita médica a través del sitio web & $2(3,8)$ & $51(96,2)$ \\
\hline El hospital está presente en alguna red social y existen enlaces a ellas desde la web & $7(13,2)$ & $46(86,8)$ \\
\hline
\end{tabular}

$\mathrm{n}$ : número de sitios web; ${ }^{a}$ : porcentaje sobre el total de sitios web estudiados 
media de 8,64 (DE 2,7) puntos, con valores máximos y mínimos de 0 y 12 puntos respectivamente.

De los 19 criterios restantes usados para evaluar esta variable "Información presentada", sólo 9 criterios fueron superados por más de la mitad de las webs, y los 10 criterios restantes no fueron superados por la mitad de las webs (Tabla III).

\section{Actualización de contenidos}

Las 53 webs estudiadas obtuvieron en "Actualización de contenidos", una media de 4,28 (DE
$3,16)$ sobre 10 puntos posibles, con valores mínimos y máximos de 0 y 10 puntos respectivamente (Tabla I).

Sólo $13(24,5 \%)$ de las webs informan sobre la fecha de actualización de contenidos. No obstante, por las fechas de publicación de los contenidos y otros indicios se ha comprobado que $30(56,6 \%)$ webs contienen información actualizada con menos de un mes desde la fecha de revisión, 19 (35,85\%) webs presentaban su información más reciente con más de seis meses sin actualizar y $4(7,55 \%)$ sitios llevaban entre 1 y 6 meses sin actualizar (Tabla IV).

Tabla III. Resultados de la variable "Información presentada" en el sitio web

\begin{tabular}{|c|c|c|}
\hline \multirow{2}{*}{ Criterios } & Sí lo cumple & No lo cumple \\
\hline & $n(\%)^{a}$ & n $(\%)^{a}$ \\
\hline Hay información de acceso al hospital (dirección y/o mapa de situación) & $53(100)$ & $0(0)$ \\
\hline Aparece dirección y/o mapa del hospital (edificios, plantas, consulta) & $37(69,8)$ & $16(30,2)$ \\
\hline Existe presentación del hospital (carta del gerente, visión, misión, etc.) & $50(94,3)$ & $3(5,7)$ \\
\hline Identificación del personal directivo con organigrama y nombres & $35(66)$ & $18(34)$ \\
\hline Identifican algún personal médico y de enfermería con cargo y nombre & $16(30,2)$ & $37(69,8)$ \\
\hline Datos de la actividad asistencial por servicios del hospital & $10(18,9)$ & $43(81,1)$ \\
\hline Datos de la actividad la asistencial en la memoria general del hospital & $35(66)$ & $18(34)$ \\
\hline Datos de resultados (mortalidad, infecciones nosocomiales, etc.) & $2(3,8)$ & $51(96,2)$ \\
\hline $\begin{array}{l}\text { Existe información epidemiológica de interés para el hospital y la población (brote de } \\
\text { legionella, gripe aviar, etc.) }\end{array}$ & $2(3,8)$ & $51(96,2)$ \\
\hline Existe cartera de servicios del hospital por especialidades & $15(28,3)$ & $38(71,7)$ \\
\hline Existe cartera de servicios general del hospital & $38(71,7)$ & $15(28,3)$ \\
\hline Hay información sobre listas de espera & $3(5,7)$ & $50(94,3)$ \\
\hline Existe sección sobre consejos de salud & $13(24,5)$ & $40(75,5)$ \\
\hline Existe información sobre preparación para pruebas diagnósticas & $3(3,8)$ & $51(96,2)$ \\
\hline Hay información sobre enfermedades o enlace a páginas que la ofrezca & $16(30,2)$ & $37(69,8)$ \\
\hline Información para el usuario sobre actividades del hospital & $29(54,7)$ & $24(45,3)$ \\
\hline Sección con noticias del hospital o de interés general & $46(86,8)$ & $7(13,2)$ \\
\hline Sección de prensa o de comunicación del hospital & $22(41,5)$ & $31(58,5)$ \\
\hline Existe apartado dedicado a la investigación y/o docencia-formación & $48(90,6)$ & $5(9,4)$ \\
\hline \multicolumn{3}{|l|}{ Guía para el paciente } \\
\hline Hay información sobre cómo llegar al hospital (medios de transporte) & $42(79,2)$ & $11(20,8)$ \\
\hline Hay información de horario de visitas y llamadas & $39(73,6)$ & $14(26,4)$ \\
\hline Hay información sobre derechos y deberes del usuario & $49(92,5)$ & $4(7,5)$ \\
\hline Hay información sobre el Servicio Atención al Usuario y ubicación & $39(73,6)$ & $14(26,4)$ \\
\hline Hay información sobre cafetería, horario, ubicación y máquinas refresco & $24(45,3)$ & $29(54,7)$ \\
\hline Hay información sobre cómo realizar una reclamación o sugerencia & $36(67,9)$ & $17(32,1)$ \\
\hline
\end{tabular}

Tabla IV. Resultados de las variables "Actualización de los contenidos", "Referencias de calidad", "Información para profesionales" e "Información para proveedores"

\begin{tabular}{|c|c|c|}
\hline \multirow{2}{*}{ Variables y criterios } & Sí lo cumple & No lo cumple \\
\hline & $n(\%)^{a}$ & $n(\%)^{a}$ \\
\hline \multicolumn{3}{|l|}{ Actualización de los contenidos } \\
\hline Existe fecha de actualización & $13(24,5)$ & $40(75,5)$ \\
\hline Información actualizada menos de 1 mes desde la fecha de revisión & $30(56,6)$ & $23(43,4)$ \\
\hline \multicolumn{3}{|l|}{ Referentes de calidad } \\
\hline $\begin{array}{l}\text { Webs adheridas a algún sello acreditador de calidad específico para webs de Salud (Hon } \\
\text { Code, WMA, ACSA) }\end{array}$ & $4(7,55)$ & $49(92,45)$ \\
\hline \multicolumn{3}{|l|}{ Información para profesionales } \\
\hline Existe apartado específico para el profesional & $36(67,9)$ & $17(32,1)$ \\
\hline \multicolumn{3}{|l|}{ Información para proveedores } \\
\hline Existe apartado específico para proveedores & $35(66)$ & $18(34)$ \\
\hline
\end{tabular}

$\mathrm{n}$ : número de sitios web; ${ }^{a}$ : porcentaje sobre el total de sitios web estudiados 


\section{Referentes de calidad}

Las 53 webs evaluadas obtuvieron en referentes de calidad una media de 0,75 (DE 2,67) puntos sobre 10 puntos posibles, con valores mínimos y máximos de 0 y 10 puntos respectivamente (Tabla I).

Del total de las webs evaluadas sólo 4 (7,55\%) estaban adheridas a algún sello acreditador de calidad específico para webs de Salud, pues 2 webs $(3,77 \%)$ estaban acreditadas por la Agencia de Calidad Sanitaria de Andalucía, en una web $(1,88 \%)$ figuró el sello de la Web Médica Acreditada (WMA), y también en otra web $(1,88 \%)$ se acreditó el código Hon Code (Tabla IV).

\section{Información para el profesional y proveedores}

Las medias aritméticas y las desviaciones obtenidas en las variables "Información para profesionales" e "Información para proveedores" (Tabla I), son acordes al hecho de que las dos terceras partes de las webs evaluadas presentan información en apartados o secciones específicas para los profesionales y proveedores del hospital (Tabla IV).

\section{DISCUSIÓN}

El objetivo de esta investigación fue evaluar la calidad y describir las características de los sitios web de los grandes hospitales españoles. De los resultados obtenidos, destaca primeramente que casi un tercio de los principales hospitales españoles carecían de sitio web, lo cual coincide con los trabajos de Arencibia-Jiménez y Aibar-Remón (2007), y de Díaz y Chaín (2008), que concluyeron que "un número muy elevado de hospitales en España, no poseen sitio web". Esto llama poderosamente la atención porque inmersos en la sociedad de la información y del conocimiento, y de la revolución de la Web 2.0, esta carencia resulta inapropiada para los que se supone que son los principales hospitales de España. Obviamente, todo hospital, independientemente de su tamaño, debería tener un sitio web de calidad porque, actualmente, no solo se utiliza Internet para buscar información sobre temas de salud, si no que muchas personas necesitadas de atención hospitalaria, también acceden a Internet para buscar el hospital adecuado a la asistencia sanitaria que precisan (Rafe y Monfaredzadeh, 2012).

Al evaluar la calidad de las webs de hospitales, resultó relevante conocer que la puntuación media de las webs analizadas (126,6 puntos), no superaba la mitad de la máxima puntuación posible (130 puntos). Según este dato, y a la luz del criterio establecido en este trabajo, coincidente con el de Conesa y otros (2011), se puede afirmar que, en general, la calidad de las webs de los hospitales españoles de gran tamaño es baja. Estos resultados y su interpretación coinciden con los trabajos de Mira y otros (2006), sobre la calidad de las webs de los hospitales públicos españoles, y con los de Díaz y
Chaín (2008), que estudiaron las webs de los hospitales de referencia españoles y concluyeron que "la calidad del contenido de las webs analizadas es insuficiente" y, por tanto, "claramente mejorable". Otro trabajo, similar al nuestro pero con población y procedimiento metodológico distinto (ArencibiaJiménez y Aibar-Remón, 2007), también coincide con nuestro estudio, pues esos autores obtienen como conclusión que la calidad de las webs de los hospitales españoles es baja. Igualmente, Conesa y otros (2011) evaluaron la calidad de las webs de información de salud, y aunque es más difícil hacer comparaciones con nuestra investigación, conviene reseñar que también destacaron una baja calidad de las webs sanitarias analizadas.

De las 53 webs valoradas en este trabajo, sólo 22 $(28,21 \%)$ superaban la mitad de la máxima puntuación posible (130 puntos) y, por tanto, estas son las que se pueden considerar webs de relativa calidad (Anexo III). Entre esas webs de mayor calidad figuran las de los Hospitales Reina Sofía (Córdoba), Vall D'Hebron (Barcelona), Virgen de las Nieves (Granada), Carlos Haya (Málaga), Complejo Hospitalario de Albacete y la Fundación Jiménez Díaz (Madrid). Estos sitios webs también coinciden en ocupar los puestos más altos en la clasificación de calidad de webs de hospitales españoles, realizada por Díaz y Chaín (2008).

Los sitios web de mayor calidad de nuestro estudio quedan muy alejados de la máxima puntuación posible que podía obtener una web (260 puntos). Baste señalar que las 13 webs que estaban por encima del valor del tercer cuartil (143,9 puntos), consiguieron entre 144,2 y 175,6 puntos (Anexo III), lo cual queda muy distante de esos 260 puntos máximos y confirma que las webs de hospitales evaluadas son, en general, de baja calidad.

Según las puntuaciones medias obtenidas en las ocho variables estudiadas se puede afirmar que las webs analizadas se caracterizan positivamente por su accesibilidad, usabilidad y por ofrecer información y servicios específicos a profesionales y proveedores, pues en cada una de estas cuatro variables se supera la mitad de la máxima puntuación posible (Tabla I). Esto evidencia el interés de los grandes hospitales españoles por facilitar el acceso y navegación en sus webs, tanto a usuarios como a profesionales y proveedores. No obstante, a pesar de esa buena puntuación media en la variable "Accesibilidad", resultó que más de la mitad de las webs de hospitales españoles de gran tamaño no cumplen ningún criterio WAI de accesibilidad y poco más de la cuarta parte de las webs evaluadas sólo cumplen el nivel mínimo de accesibilidad exigible para cualquier web (WAI-A). En consecuencia, las webs evaluadas y, sobre todo, las 27 que no cumplen ningún criterio de accesibilidad, deben incrementar sus niveles WAI para que sus contenidos y servicios estén disponibles para cualquier persona con independencia de sus capacidades visuales, auditivas, cognitivas o motrices, y de for- 
ma que la información pueda ser presentada en las webs, con independencia de los equipos informáticos y aplicaciones utilizados por los usuarios para su acceso (Ramos y Clabo, 2008).

Otros trabajos que estudiaron la accesibilidad a las webs de hospitales obtuvieron peores resultados que los nuestros. Así, Mira y otros (2006) concluyeron que ninguno de los sitios web de los hospitales públicos españoles que evaluaron, cumplían los requisitos necesarios para ser considerados accesibles. Por tanto, el mejor nivel de accesibilidad de las webs de hospitales obtenido en esta investigación respecto a lo concluido por Mira y otros (2006), puede obedecer a que seis años después de aquel estudio, los sitios webs de hospitales españoles han mejorado sensiblemente su calidad y ahora son más accesibles.

Muchos de los estudios realizados sobre calidad de los sitios webs se han centrado exclusivamente en analizar la accesibilidad y la usabilidad (Ramos y Clabo, 2008; Jiménez y otros, 2009b; Doblas, 2007; Mira y otros, 2006). Según esto, se podría entender erróneamente que por el simple hecho de satisfacer únicamente esas dos características -accesibilidad y usabilidad-, se consiguen unas webs de calidad. Por el contrario, además de facilidad en el acceso y en su manejo, las webs de calidad deben satisfacer otras características como poseer herramientas para interactuar con los usuarios y facilitar información útil, eficaz y actualizada, tanto a los profesionales como a los usuarios del hospital.

En nuestra investigación, las puntuaciones medias de las variables "Interactividad", "Información presentada", "Actualización de contenidos" y "Referentes de calidad", no alcanzaban la mitad de la puntuación máxima para cada una de ellas. (Tabla I). Estas variables se consideran fundamentales para valorar la calidad de los sitios web, recibiendo una alta puntuación dentro del esquema de evaluación, por lo que tienen una influencia sustancial en la puntuación final de las webs analizadas.

En este mismo sentido, Díaz y Chaín (2008) también obtuvieron resultados semejantes a los nuestros pues las webs que evaluaron no reunían los criterios mínimos de calidad exigibles para que el ciudadano se informe, consulte datos y consejos básicos, o interactúe con el sitio web, porque la mayoría de esas webs no facilitaban la interacción con los pacientes ni presentaban información suficiente para estos. También los resultados de ArencibiaJiménez y Aibar-Remón (2007), coinciden con los nuestros en cuanto a la escasez de herramientas para interactuar con los usuarios, deficiente información presentada y poca adherencia de las webs de hospitales a sellos de calidad de salud.

El déficit en "Interactividad y relación con los usuarios" de las webs analizadas se debe a la casi total ausencia de herramientas para interactuar y relacionarse a través de las webs con los usuarios de los hospitales. Este déficit llama la atención por- que inmersos en la Web 2.0, con funcionalidades enfocadas al usuario final de Internet que generan colaboración y servicios, aún existe una práctica imposibilidad para que los ciudadanos puedan pedir cita médica desde las webs. También destaca el hecho de que un tercio de sitios webs de hospitales carezcan de buzón de sugerencias o que casi la mitad de los hospitales no faciliten emails para contactar con el servicio de atención a los usuarios (Tabla II). Estos resultados coinciden también con los de Doblas (2007), que afirma que las webs de los hospitales universitarios españoles aún no han desarrollado servicios que vayan más allá de la información, como, por ejemplo, la realización de gestiones online, la publicación de contenidos creados por usuarios o la personalización de la información (Rodríguez-Martínez y otros, 2012). La ausencia de estas funcionalidades básicas de interrelación obliga a los hospitales a plantearse la necesidad de incorporarlas en sus webs y contribuir así a evitar desplazamientos innecesarios al hospital y gasto de tiempo, tanto a usuarios como a profesionales.

En ese déficit de "Interactividad y relación con los usuarios" observado en las webs evaluadas también influye la bajísima presencia de los hospitales españoles en las redes sociales, dado que sólo 7 de los 53 hospitales estudiados están presentes en algún medio social, como Twitter, Facebook, You Tube, Flickr o en blogs. En Estados Unidos, Luxemburgo o Alemania, la tendencia es la contraria pues los hospitales están tomando conciencia de los beneficios que los medios sociales pueden ofrecer y los utilizan cada vez más (Van de Belt y otros, 2012). En consecuencia, los grandes hospitales españoles no aprovechan actualmente las ventajas que ofrece la Web 2.0, y más concretamente las redes sociales, para posibilitar una mejor interacción web-usuarios, lo que denota la escasa conciencia sobre los beneficios que los medios sociales pueden ofrecer a unos usuarios y pacientes que cada vez usan más las redes sociales.

Por el contrario, la presencia activa de los hospitales en las redes sociales podría convertir a los usuarios en unos agentes más participativos y comprometidos con los objetivos de salud y tender a una atención cada vez más centrada en el paciente, lo cual podría repercutir en la calidad de los cuidados profesionales de salud. Por ello, los hospitales deberían considerar los beneficios que las redes sociales pueden ofrecer y usarlos cada vez más, para mejorar la interacción y la comunicación con profesionales, usuarios y familiares (Van de Belt y otros, 2012).

En nuestro estudio destaca la preocupación de los hospitales españoles de gran tamaño por disponer en sus webs de buenas guías con información general para pacientes, siendo este criterio superado por gran parte de las webs (Tabla III). Esto indica que los hospitales estudiados se interesan por presentar información básica para los usuarios, como su 
ubicación, acceso a sus instalaciones, horario de visitas o ubicación de la cafetería. También muestran interés por facilitar información sobre los derechos y deberes de los usuarios, sobre el servicio de atención a los usuarios y cómo realizar reclamaciones o sugerencias (Tabla III). Estas conclusiones son contrarias a las de Díaz y Chaín (2008), lo cual puede ser explicado porque en los cuatro años transcurridos entre ese estudio y el nuestro, probablemente los hospitales han incrementado su interés por facilitar buenas guías de servicios complementarios a los usuarios y familiares.

En las webs evaluadas no se suele identificar correctamente a los profesionales sanitarios que ejercen en los hospitales, y hay una casi total ausencia de información para los usuarios sobre temas de salud generales y especializados. Tampoco se informa sobre las listas de espera ni sobre la preparación que deben seguir los pacientes antes de una prueba diagnóstica, y llama la atención que sólo una cuarta parte de las webs contengan información sanitaria (Tabla III). Díaz y Chaín (2007) también señalan estos déficits en su estudio, destacando que si los hospitales no ofrecen ningún tipo de actividad asistencial en sus webs, es decir, no disponen de consejos de salud, no divulgan temas sobre prevención, ni aclaran conceptos sobre enfermedades crónicas que pudiesen reducir las dudas de los usuarios, esas webs no contribuyen a reducir el número de consultas presenciales, sobre todo de enfermos crónicos. En estos casos, si los usuarios dispusiesen de información de este tipo en las webs de hospitales, además de demandar menos consultas presenciales con médicos y enfermeras, podrían implicarse más en sus autocuidados y mejora de la salud. Si además, se identificase correctamente a los profesionales sanitarios en las webs de los hospitales y se pudiese interactuar con ellos a través del sitio web para posibles aclaraciones sobre temas de salud, se reduciría también la presencialidad de los usuarios en las consultas hospitalarias para solventar estos temas.

También hay que señalar que aunque la mayoría de hospitales presenta su cartera de servicios general, menos de la tercera parte de hospitales presentan la cartera de servicios por especialidades (Tabla III). A este respecto, y como señalan Díaz y Chaín (2007), es necesario que los hospitales informen sobre qué servicios especializados y actividades se realizan en ellos, así como de los datos de la actividad asistencial y de los índices de resultados de dicha actividad, siendo la web el lugar idóneo para obtener esa información. Conocer el éxito asistencial de un hospital y de sus profesionales, es fundamental para los usuarios y constituye una información muy apreciable cuando una persona busca una segunda opinión médica o quiere hacer uso de la libre elección de médico especialista, por lo que las webs de hospitales deberían incluir la cartera de servicios por especialidades, sus datos y resultados de la actividad asistencial.
Igualmente, llama la atención que más de la mitad de los grandes hospitales españoles carezcan en sus sitios webs de secciones para el gabinete de prensa o de comunicación, lo cual indica que la gran mayoría de esos hospitales desatiende o no es prioritario para ellos relacionarse con los medios de comunicación social a través de Internet (Tabla III).

Sobre la actualización de los contenidos de las webs, los hospitales deben esforzarse por mantenerlas al día, pues más de las tres cuartas partes de webs carecían de fecha de actualización y casi la mitad de ellas llevaban más de un mes sin actualizar (Tabla IV). Esa falta de actualización reduce la credibilidad y utilidad de la información ofrecida en las webs de los hospitales evaluados, por lo cual estos deberían esforzarse en el cumplimiento de estos criterios.

En cuanto a los referentes de calidad presentes en las webs de los hospitales, destaca la casi nula adhesión de esas webs a algún sello o código de calidad específico para sitios web sanitarios (Tabla IV). Aunque el cumplimiento de este criterio podría ser de importancia relativa, dado que los sellos de calidad son más propios de webs sobre temas de salud o medicina que de hospitales, no se debe olvidar que los hospitales son instituciones encargadas de la prestación de asistencia sanitaria especializada, que deben asegurar y acreditar una alta calidad, reconocida por organismos externos, tanto asistencial como organizacional, lo que también debería incluir a sus espacios web. En este sentido, sería conveniente que las webs de hospitales se adhiriesen a códigos y normas de calidad específicas para webs sanitarias, pues estos son recomendaciones y criterios de calidad propuestos para el desarrollo y el contenido de los sitios web, que sirven como una herramienta de autoevaluación para los proveedores de las webs y cumplen una función educativa tanto para esos proveedores como para los usuarios (Azpilicueta y otros, 2007).

Las fortalezas de esta investigación estriban en que se ha completado y actualizado el cuestionario confeccionado por Arencibia-Jiménez y Aibar-Remón (2007), para medir la calidad de las webs de hospitales, y que además, es un estudio sin sesgo de subjetividad y carente de posible variación intraoperador en la recogida de datos, al ser su autor el que evaluó todas las webs. Este trabajo, como todos los que evalúan sitios webs, tiene dos limitaciones debidas a la propia naturaleza dinámica y cambiante de Internet: los resultados de este estudio tendrían una validez temporal pues una página web puede ser incorporada, retirada o modificada en cualquier momento (Jiménez y otros, 2009a; Navarro-Arnedo, 2009). La segunda limitación es que algunos elementos que se evalúan en las webs, a veces no son fácilmente apreciables debido a la falta de normalización o acuerdo sobre dónde deben aparecer, por lo que no es posible poder garantizar de forma absoluta la ausencia de esos elementos en una web (Navarro-Arnedo, 2009). No 
obstante, creemos que estas limitaciones no afectan a la validez externa de nuestro trabajo porque, como ya se ha comentado, sus resultados coinciden en gran medida con investigaciones similares.

Unas webs de hospitales con calidad son fundamentales para ofrecer mejores servicios a los usuarios que redunden en mayores niveles de salud (Rafe y Monfaredzadeh, 2012), por lo que es recomendable que observen los criterios establecidos internacionalmente para garantizar la calidad de las webs sanitarias (Domínguez-Castro e Iñesta-García, 2004). Por ello, la aplicación práctica de esta investigación reside en que sus resultados deberían considerarse como una oportunidad de mejora, que invite a los gestores de los hospitales a implementar una mejor práctica en el desarrollo de sus webs para aumentar su calidad, subsanando aquellas características deficitarias de las webs e incorporando nuevas herramientas que faciliten más información sobre salud, y una mejor y mayor relación de los ciudadanos con los servicios y profesionales del hospital, a través de sus webs. Incrementando así su calidad, las webs de hospitales serían más útiles y confiables para los usuarios (Domínguez-Castro e Iñesta-García, 2004), y favorecerían la atención sanitaria de la población. Además, se conseguiría una mejor gestión y administración sanitaria, al reducir la presencialidad del usuario en el hospital, los costes y trámites, contribuyendo a lograr hospitales y un sistema sanitario más eficaz, sostenible y eficiente.

\section{CONCLUSIONES}

En este estudio descriptivo, donde se evaluó la calidad y características de las webs de los 78 hospitales españoles con más de 500 camas, se concluye que un tercio de esos hospitales carecen de sitio web y que en general, la calidad de sus webs es baja. Esto implicaría que esos grandes hospitales, centrados en conseguir para los ciudadanos la mejor atención sanitaria especializada, habrían descuidado la comunicación mediante las nuevas tecnologías. En definitiva, los hospitales deberían avanzar hacia el importante objetivo de interrelación con la sociedad mediante webs de calidad.

Más específicamente, se puede afirmar que las webs estudiadas se caracterizan positivamente por ser accesibles, usables y por presentar información para el profesional y para proveedores, a la vez que son deficitarias en interactividad y de muy pobre presencia en los medios sociales, por lo que no facilitan la interacción y la relación con los usuarios. A la luz de estos resultados se puede concluir también que los hospitales españoles evaluados siguen considerando a los sitios webs como espacios meramente informativos, concebidos para presentar información, lo que justifica su interés en lograr una buena accesibilidad y un fácil manejo de las webs.
Con esta consideración, las webs de los hospitales analizados se conciben aún como espacios estáticos pensados para la comunicación unilateral y no dialógica, donde se privilegia un proceso unidireccional de transferencia de información, que no facilitan los procesos de intercambio y que reducen a su audiencia a ejercer un rol pasivo en el proceso comunicativo (Fenoll, 2011). De este modo, las webs de los grandes hospitales españoles quedan muy alejadas de las posibilidades hipertextuales e interactivas de los actuales cibermedios que permiten la interacción del público a la vez que fomentan su participación y colaboración (Fenoll, 2011). En la práctica, los usuarios de las webs de esos hospitales siguen siendo considerados como simples consumidores y receptores pasivos de información.

En definitiva, para que los sitios webs sean de calidad deben ser accesibles y usables, y además han de acompañarse de herramientas que posibiliten interactuar con los usuarios y que provean información útil y eficaz, tanto al público interno como externo del hospital.

\section{BIBLIOGRAFÍA}

Agencia de Calidad Sanitaria de Andalucía. (2012). Certificación de Páginas Web Sanitarias. Sevilla: Consejería de Salud de la Junta de Andalucía. http://www.juntadeandalucia.es/ agenciadecalidadsanitaria/es/certificacion/paginasweb/index.html [19 de febrero de 2014].

Arencibia-Jiménez, M.; Aibar-Remón, C. (2007). Páginas web de hospitales. Realidad virtual o... ¿son realmente un medio útil para la difusión de información para sus usuarios? Revista de Calidad Asistencial, vol. 22 (3), 118-127. http://dx.doi. org/10.1016/S1134-282X(07)71206-X

Azpilicueta, I.; Bermúdez, C.; Silva, M.M.; Valverde, I.; Martiarena, A.; García, J.F.; Faus, M.J. (2007). Adecuación a los códigos de conducta para información biomédica en Internet de sitios web útiles para el seguimiento farmacoterapéutico. Gaceta Sanitaria, vol. 21 (3), 204-209. http:// dx.doi.org/10.1157/13106802

Bermúdez-Tamayo, C.; Jiménez-Pernett, J.; García Gutiérrez, J.F.; Azpoñocieta Cengotitobengoa, I.; Milena Silva-Castro, M.; Babio, G.; Plazaola Castaño, J. (2006). Cuestionario para evaluar sitios web sanitarios según criterios europeos. Atención Primaria, vol. 38 (5), 268-274. http://dx.doi. org/10.1157/13092985

Chaín, C.; Muñoz, A.; Más, A. (2008). La gestión de información en las sedes web de los ayuntamientos españoles. Revista Española De Documentación Científica, vol. 31 (4), 612-638.

Conesa, M.C.; Aguinaga, E.; Hernández, J.J. (2011). Evaluación de la calidad de las páginas web sanitarias mediante un cuestionario validado. Atención Primaria, vol. 43 (1), 33-40. http:// dx.doi.org/10.1016/j.aprim.2010.01.007, PMid:20304528. 
Conesa, M.C.; Aguinaga, E. (2009). Evaluación de la calidad de las páginas web con información sanitaria: una revisión bibliográfica. bid Textos universitaris de biblioteconomia i documentació, 23. http://www.ub.edu/bid/23/conesa2.htm [7 de julio de 2012].

Díaz, A; Chaín, C. (2008). Contenidos de las Web de los hospitales españoles: un aspecto pendiente de discusión y unificación. Acimed, vol. 17(1). http:// bvs.sld.cu/revistas/aci/vol17 1 08/aci04108.htm [10 de marzo de 2007].

Doblas, S. (2007). Usabilidad de sitios Web de hospitales universitarios españoles. Evaluación Heurística. En: Ponencias al XI Congreso Nacional de Internet, Telecomunicaciones y Sociedad de la Información. Málaga. 14 a 16 de mayo de 2007. http://www.informatica2007.sld.cu/ Members/sdoblas/evaluacion-heuristica-delos-sitios-web-de-hospitales-universitariosespanoles/2006-12-04.7973867127/download [19 de febrero de 2014].

Domínguez-Castro, A.; Iñesta-García, A. (2004). Evaluación de la calidad de las webs de centros de farmacoeconomía y economía de la salud en Internet mediante un cuestionario validado. Gaceta Sanitaria, vol. 18 (4), 295-304. http://dx.doi. org/10.1016/S0213-9111(04)72016-6

European Commission (2013). Europe's Information Society Thematic Portal. eHealth Action Plan 2012-2020: Innovative healthcare for the 21st century http://ec.europa.eu/information_society/ newsroom/cf/itemdetail.cfm?item_id=9156 [13 de abril de 2013].

Fenoll, V. (2011). Usuarios activos y pasivos. La interactividad de la Audiencia en los medios digitales. El caso de la fórmula 1 en Valencia. Aposta. Revista de ciencias sociales, 51, 26 p. http://www. apostadigital.com/revistav3/hemeroteca/fenoll.pdf [1 de junio de 2013].

Jiménez, J.; García, J.F.; Bermúdez, C.; Silva M.M.; Tuneu, L. (2009a). Evaluación de sitios web con información sobre medicamentos. Atención Primaria, vol. 41 (7), 360-366. http://dx.doi.org/10.1016/j. aprim.2008.10.009, PMid:19464077.

Jiménez, J.; García, J.F.; Bermúdez, C. (2009b). Accesibilidad de sitios web sobre salud para mayores. Revista Española de Geriatría y
Gerontología, vol. 44 (6), 342-348. http://dx.doi. org/10.1016/j.regg.2009.07.001, PMid:19926357.

Ministerio de Sanidad, Servicios Sociales e Igualdad. (2012). Catálogo Nacional de Hospitales 2012. Madrid: Ministerio de Sanidad, Servicios Sociales e Igualdad, p. 15. http://www.msc.es/ciudadanos/ prestaciones/centrosServiciosSNS/hospitales/ docs/CNH2012.pdf [7 de julio de 2012].

Mira, J.J.; Llinas, G.; Tomas, O.; Pérez-Jover, V. (2006). Quality of websites in Spanish public hospitals. Medical Informatics and the Internet in Medicine, vol. 31 (1), 23-44.

Navarro-Arnedo, J.M. (2009). Análisis de las páginas web de las Unidades de Cuidados Intensivos de España. Enfermería Intensiva, vol. 20 (4), 148-158. http://dx.doi.org/10.1016/S1130-2399(09)73223-7

Navarro-Arnedo J.M. (2008). Enfermería en las páginas web de los hospitales españoles. Metas de Enfermería, vol. 11 (8), 27-32.

Rafe, V.; Monfaredzadeh, M. (2012). A qualitative framework to assess hospital/medical websites. Journal of Medical Systems, vol. 36 (5), 29272939. http://dx.doi.org/10.1007/s10916-0119771-5, PMid:21874337.

Ramos, I.; Clabo, N. (2008). Calidad de las sedes web de las Oficinas de Transferencia de Resultados de Investigación universitarias andaluzas: contenidos, usabilidad y accesibilidad. Revista Española de Documentación Científica, vol. 3 1(3), 366-395.

Rodríguez-Martínez, R.; Codina, L; Pedraza-Jiménez, R. (2012). Indicadores para la evaluación de la calidad en cibermedios: análisis de la interacción y de la adopción de la Web 2.0. Revista Española de Documentación Científica, vol. 35 (1), 61-93. http://dx.doi.org/10.3989/redc.2012.1.858

Van de Belt, T.H.; Berben, S.A.A.; Samsom, M.; Engelen, L.J.; Schoonhoven, L. (2012). Use of social media by Western European hospitals: Iongitudinal study. Journal of Medicine Internet Research, vol. 14(3), e61. http://www.jmir.org/2012/3/e61/ [8 de noviembre de 2012]. http://dx.doi.org/10.2196/ jmir.1992, PMid:22549016 PMCid:PMC3799605.

Web Médica Acreditada (2012). ¿Qué es Web Médica Acreditada? Barcelona: Colegio Oficial de Médicos de Barcelona. http://wma.comb.es/es/usuariosque-es-wma.html [20 de julio de 2012]. 


\section{Anexo I}

Hospitales españoles con más de 500 camas y su presencia en la Web*

\begin{tabular}{|c|c|c|c|}
\hline 1 & H G U Gregorio Marañón (Madrid) & 40 & Complejo Hospitalario de Jaén \\
\hline 2 & Complejo Hospitalario U de Santiago de Compostela & 41 & Complejo Hospitalario de Toledo \\
\hline 3 & H U Virgen del Rocío (Sevilla) & 42 & H Clínico U de Valladolid \\
\hline 4 & H U i Politécnic la Fe de Valencia & 43 & $\begin{array}{l}\text { Complex Asistencial en Salut Mental Benito Menni } \\
\text { (Sant Boi de Llobregat) }\end{array}$ \\
\hline 5 & Complexo Hospitalario U A Coruña & 44 & H U Puerta del Mar (Cádiz) \\
\hline 6 & H U 12 de Octubre (Madrid) & 45 & H U de Canarias (Tenerife) \\
\hline 7 & H U Reina Sofía (Córdoba) & 46 & Fundación Jiménez Díaz (Madrid) \\
\hline 8 & Complejo U La Paz (Madrid) & 47 & H de Basurto (Bilbao) \\
\hline 9 & H U Miguel Servet (Zaragoza) & 48 & Complejo Hospitalario U de Albacete \\
\hline 10 & H U Central Asturias & 49 & Institut Pere Mata (Reus) \\
\hline 11 & Complejo Hospitalario U de Vigo & 50 & H U Virgen de la Victoria (Málaga) \\
\hline 12 & Hospitals Vall D'Hebron (Barcelona) & 51 & H Santa Creu i Sant Pau (Barcelona) \\
\hline 13 & H Regional U Carlos Haya (Málaga) & 52 & H U Rio Hortega (Valladolid) \\
\hline 14 & H Donostia & 53 & H U San Cecilio (Granada) \\
\hline 15 & H U Ramón y Cajal (Madrid) & 54 & H Juan Ramón Jiménez (Huelva) \\
\hline 16 & Complejo Hospitalario de Navarra & 55 & Centro San Juan de Dios (Ciempozuelos) \\
\hline 17 & H U Virgen de las Nieves (Granada) & 56 & Complejo H U de Cartagena \\
\hline 18 & H U Virgen Macarena (Sevilla) & 57 & Complejo H de Pontevedra \\
\hline 19 & H U de Bellvitge (Hospitalet de Llobregat) & 58 & H U de Getafe \\
\hline 20 & Complejo Asistencial U de León & 59 & H U Puerta de Hierro (Madrid) \\
\hline 21 & Complejo H U de Badajoz & 60 & H U Virgen de Valme (Sevilla) \\
\hline 22 & H Clínico San Carlos (Madrid) & 61 & H U Príncipe de Asturias (Alcalá de Henares) \\
\hline 23 & C Asistencial U de Salamanca & 62 & Complejo Asistencial de Zamora \\
\hline 24 & H Clinic Provincial de Barcelona & 63 & Hospital Psiquiatric (Salt) \\
\hline 25 & H U de Cruces (Baracaldo) & 64 & Consorcio H G U de Valencia \\
\hline 26 & Complejo H U Insular Materno-Infantil (Las Palmas) & 65 & H Clínico Universitario (Valencia) \\
\hline 27 & Complejo H Dr. Negrín (Las Palmas) & 66 & H G de Castelló \\
\hline 28 & Complejo Asistencial U de Burgos & 67 & Complejo H San Pedro (Logroño) \\
\hline 29 & Complejo H U de Ourense & 68 & H Povisa SA Vigo \\
\hline 30 & H U Nuestra Señora de la Candelaria (Tenerife) & 69 & H G de Ciudad Real \\
\hline 31 & Complejo H Torrecardenas (Almería) & 70 & H U de la Princesa (Madrid) \\
\hline 32 & H Clínico U Virgen de la Arrixaca (Murcia) & 71 & H de Jerez \\
\hline 33 & $\mathrm{H}$ de Sabadell & 72 & H U Dr Peset (Valencia) \\
\hline 34 & Complejo H Xeral-Calde Lugo & 73 & H U Mútua de Terrassa \\
\hline 35 & H U Marqués Valdecilla (Santander) & 74 & Complejo Hospitalario de Cáceres \\
\hline 36 & H G U de Alicante & 75 & H de la Defensa Gómez Ulla (Madrid) \\
\hline 37 & Complejo Asistencial Son Espases (Illes Balears) & 76 & H G de Elda Virgen de la Salud \\
\hline 38 & $\begin{array}{l}\text { Parc Sanitari Sant Joan de Déu (Sant Boi de } \\
\text { Llobregat) }\end{array}$ & 77 & H U Germán Trias i Pujol (Badalona) \\
\hline 39 & H Clínico U Lozano Blesa (Zaragoza) & 78 & H Punta de Europa (Algeciras) \\
\hline
\end{tabular}

H: Hospital; G: General; U: Universitario

$\square$ Hospital con sitio web

*Los hospitales están ordenados de mayor a menor número de camas 
Anexo II

Cuestionario de evaluación de la calidad y características de las webs de hospitales

Hospital:

Dirección web:

Fecha de revisión: Puntuación total:

\begin{tabular}{|c|c|c|}
\hline $\begin{array}{l}\text { Variable, criterios y } \\
\text { puntuación máxima }\end{array}$ & Puntuación & Subcriterios \\
\hline $\begin{array}{l}\text { 1. Accesibilidad } \\
20 \text { puntos }\end{array}$ & & $\begin{array}{l}\text { Problemas Prioridad } 1 \text { ó A: } 12 \text { puntos menos no problemas } \times 0,4 \\
\text { Problemas Prioridad } 2 \text { ó AA: } 16 \text { puntos menos no problemas } \times 0,2 \\
\text { Problemas Prioridad } 3 \text { ó AAA: } 20 \text { puntos menos no problemas } \times 0,1\end{array}$ \\
\hline
\end{tabular}

\section{Usabilidad \\ 20 puntos}

2.1. Tiempos de descarga: 2 puntos

2. 2. Navegación intuitiva: 4 puntos
2 puntos: Si tiene una conexión de $0-5$ segundos con ADSL

1 punto: Conexión de 5-10 segundos con ADSL

0 puntos: Más de 15 segundos en conexión ADSL

\begin{tabular}{|c|c|}
\hline $\begin{array}{l}\text { 2. 3. Optimización para } \\
\text { distintos navegadores y } \\
\text { resoluciones: } \\
1 \text { punto }\end{array}$ & $\begin{array}{l}1 \text { punto: Si la web está optimizada para Internet Explorer } 8.0 \text { o superior y } \\
\text { otros navegadores similares } \\
\text { 0,5 puntos: Si la web está sólo optimizada para Internet Explorer } 8.0 \text { o } \\
\text { superior u otros } \\
0 \text { puntos: Si la web no está optimizada para Internet Explorer } 8.0 \text { o } \\
\text { superior }\end{array}$ \\
\hline $\begin{array}{l}\text { 2. 4. Herramienta } \\
\text { de búsqueda de los } \\
\text { contenidos de la web: } 5 \\
\text { puntos }\end{array}$ & $\begin{array}{l}5 \text { puntos: Si existe herramienta de búsqueda avanzada } \\
3 \text { puntos: Si existe herramienta de búsqueda (cajetín sin campos) } \\
0 \text { puntos: Si no existe herramienta de búsqueda }\end{array}$ \\
\hline $\begin{array}{l}\text { 2. } 5 \text {. Especificaciones } \\
\text { de los documentos } \\
\text { descargables: } \\
2 \text { puntos }\end{array}$ & $\begin{array}{l}2 \text { puntos: Si se indica peso, formato y tiempo de descarga o número de } \\
\text { páginas a descargar } \\
1 \text { punto: } \mathrm{Si} \text { se indica el peso y el formato de la descarga } \\
0 \text { puntos: Si no se indica el peso y el formato }\end{array}$ \\
\hline $\begin{array}{l}\text { 2. 6. Sección de links } \\
\text { externos para ampliar } \\
\text { información: } 1 \text { punto }\end{array}$ & $\begin{array}{l}1 \text { punto: Existe sección } \\
0 \text { puntos: No existe sección }\end{array}$ \\
\hline $\begin{array}{l}\text { 2. 7. Correcta } \\
\text { identificación de los } \\
\text { links: } 2 \text { puntos }\end{array}$ & $\begin{array}{l}2 \text { puntos: Se describen URL y nombre del site, y se puede acceder directamente } \\
1 \text { punto: Sólo se muestra URL y nombre del site } \\
0 \text { puntos: Sólo se muestra la URL o el nombre del site } \\
-2 \text { puntos: } \\
0 \text { puntos: Al evaluar los } 5 \text { primeros, todos son correctos } \\
-1 \text { punto: Hay un link que da error de conexión o la dirección ya no existe } \\
-2 \text { puntos: Hay más de un link que da error de conexión o la dirección ya no } \\
\text { existe }\end{array}$ \\
\hline
\end{tabular}

$\begin{array}{ll}\text { 2.8. Existe mapa del } & 3 \text { puntos: Sí } \\ \text { web: } 3 \text { puntos } & 0 \text { puntos: No }\end{array}$

4 puntos: Si mantiene todas las opciones del menú de navegación principal abiertas en el interior del canal (en todas las ventanas que se van abriendo) 0 puntos: Si no mantiene todas las opciones del menú de navegación principal abiertas en el interior del canal

esoluciones:

0 puntos: Si la web no está optimizada para Internet Explorer 8.0 o

5 puntos: Si existe herramienta de búsqueda avanzada

puntos: Si existe herramienta de búsqueda (cajetín sin campos)

contenidos de la web: 5

0 puntos: Si no existe herramienta de búsqueda

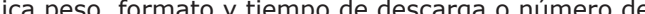
a descargar

punto: Si se indica el peso y el formato de la descarg

de los documentos

descargables:

6. Sección de links externos para amplia información: 1 punto 
Anexo II

Cuestionario de evaluación de la calidad y características de las webs de hospitales

\begin{tabular}{ll}
\hline $\begin{array}{l}\text { Variable, criterios y } \\
\text { puntuación máxima }\end{array}$ & Puntuación \\
\hline $\begin{array}{l}\text { 3. Interactividad } \\
\text { y relación con los } \\
\text { usuarios } \\
50 \text { puntos }\end{array}$ & \\
\hline $\begin{array}{l}\text { 3.1. E-mail de consulta/ } \\
\text { solicitud de información } \\
\text { general (Servicio de }\end{array}$ & 5 puntos: Sí \\
Admisión o Atención al \\
paciente): 5 puntos
\end{tabular}

3.2. Mención de

otras alternativas de

contactos/información

general (Servicio de

Admisión o Atención al

Paciente): 5 puntos
5 puntos: Si facilitan la dirección física y un teléfono y/o fax al que dirigirse de contacto

3 puntos: Si el único medio de contacto es una dirección física

0 puntos: Si no hay posibilidad de contacto
3.3. E-mail de consulta/ solicitud de información

con los servicios del

hospital: 5 puntos
5 puntos: Sí

0 puntos: No hay servicio e-mail
3.4. Mención de otras alternativas de contacto con los servicios del hospital: 5 puntos
5 puntos: Si facilitan la dirección física y un teléfono y/o fax al que dirigirse 2 puntos: Si el único medio de contacto es una dirección física

1 punto: Si existe dirección o posibilidad de contacto con algún servicio, pero no es posible contactar con todos

0 puntos: Si no hay ni posibilidad de contacto con los servicios del hospital
3.5. Existencia de buzón de sugerencias: 10 puntos
10 puntos: Existe buzón de sugerencias

0 puntos: No existe

3.6. Posibilidad de pedir cita a través de la web: 10 puntos

10 puntos: Existe la posibilidad de pedir cita a través de la web

0 puntos: No existe la posibilidad de pedir cita a través de la web

3.7. Presencia del

hospital en alguna red

social y hay enlaces a

ellas: 10 puntos
10 puntos: El hospital está presente en al menos una red social y desde la web existen enlaces a ellas

0 puntos: El hospital no está presente en redes sociales

\section{Información \\ presentada}

120 puntos

\begin{tabular}{ll}
\hline & 4 puntos: Existe dirección y mapa de situación del hospital \\
$\begin{array}{l}\text { 4.1. Acceso al hospital: } \\
4 \text { puntos }\end{array}$ & $\begin{array}{l}\text { 0 puntos: Existe sólo dirección } \\
\text { encuentra }\end{array}$ \\
\hline & \\
$\begin{array}{l}\text { 4.2. Mapa del hospital: } \\
\text { 2 puntos }\end{array}$ & 0 puntos: Hay mapa o plano del hospital (edificios, plantas, consultas, etc.) \\
\end{tabular}


Anexo II

Cuestionario de evaluación de la calidad y características de las webs de hospitales

Variable, criterios y
puntuación máxima

\begin{tabular}{ll}
\hline & \\
& 2 puntos: Hay información sobre cómo llegar al hospital (autobuses, metro...) \\
& 2 puntos: Hay información de horario de visitas y llamadas \\
4.3. Guía para el & 2 puntos: Hay información sobre derechos y deberes del usuario \\
paciente: 12 puntos & 2 puntos: Hay información sobre servicio y ubicación del SIAP (Servicio \\
& Información al Usuario) \\
& 2 puntos: Hay información sobre servicio de cafetería, horario, ubicación y \\
& máquinas de refresco... \\
& 2 puntos: Hay información sobre cómo realizar una reclamación o sugerencia
\end{tabular}
4.4. Presentación del
2 puntos: Existe presentación (carta de presentación del gerente, visión, hospital: 2 puntos
misión, objetivos, historia del hospital... Cualquiera de éstos es válido)
0 puntos: No existe presentación

\subsection{Identificación personal directivo: 5 puntos}

5 puntos: Hay organigrama con nombres

3 puntos: No aparecen los nombres de todos los responsables (sólo aparece el nombre del gerente $\mathrm{y} / \mathrm{o}$ alguno más, pero no todos)

0 puntos: No aparecen los nombres de los directivos del hospital

\subsection{Identificación del personal médico y de enfermería: 10 puntos}

10 puntos: Aparecen los nombres de todos los médicos y enfermeras de cada servicio médico

5 puntos: Aparece el nombre del jefe de servicio y de la supervisora de enfermería solamente

0 puntos: No se identifica a los médicos ni enfermeras del hospital
4.7. Datos de actividad asistencial (número de consultas, operaciones, GRD, pruebas

diagnósticas, CMA): 10

puntos
10 puntos: Presentan la información por servicios

5 puntos: Presentan la información en forma de memoria de actividad (datos

agrupados o generales del hospital)

0 puntos: No hay información de actividad

\begin{tabular}{ll}
$\begin{array}{l}\text { 4.8. Datos de } \\
\text { resultados: } 5 \text { puntos }\end{array}$ & $\begin{array}{l}5 \text { puntos: Hay algún dato de resultado (mortalidad, infecciones } \\
\text { nosocomiales,...) } \\
0 \text { puntos: No hay datos de resultado }\end{array}$ \\
\hline $\begin{array}{l}\text { 4.9. Información } \\
\text { epidemiológica de } \\
\text { interés: } 5 \text { puntos }\end{array}$ & $\begin{array}{l}5 \text { puntos: Existe información epidemiológica de interés, tanto del hospital (p. } \\
\text { ej., brote de interés legionela) como otras noticias de interés ( } p \text {. ej., gripe } \\
\text { aviar) para la población } \\
0 \text { puntos: No existe información epidemiológica de interés }\end{array}$
\end{tabular}

\subsection{Cartera de}

servicios: 10 puntos
10 puntos: Existe cartera de servicios por especialidades

5 puntos: Existe cartera de servicios general del hospital

0 puntos: No existe cartera de servicios
4.11. Lista de espera: $5 \quad 5$ puntos: Hay información sobre lista de espera

puntos
0 puntos: No hay información sobre lista de espera
4.12. Consejos de salud: 5 puntos
5 puntos: Existe sección sobre consejos de salud

0 puntos: No existe sección sobre consejos de salud 
Anexo II

Cuestionario de evaluación de la calidad y características de las webs de hospitales

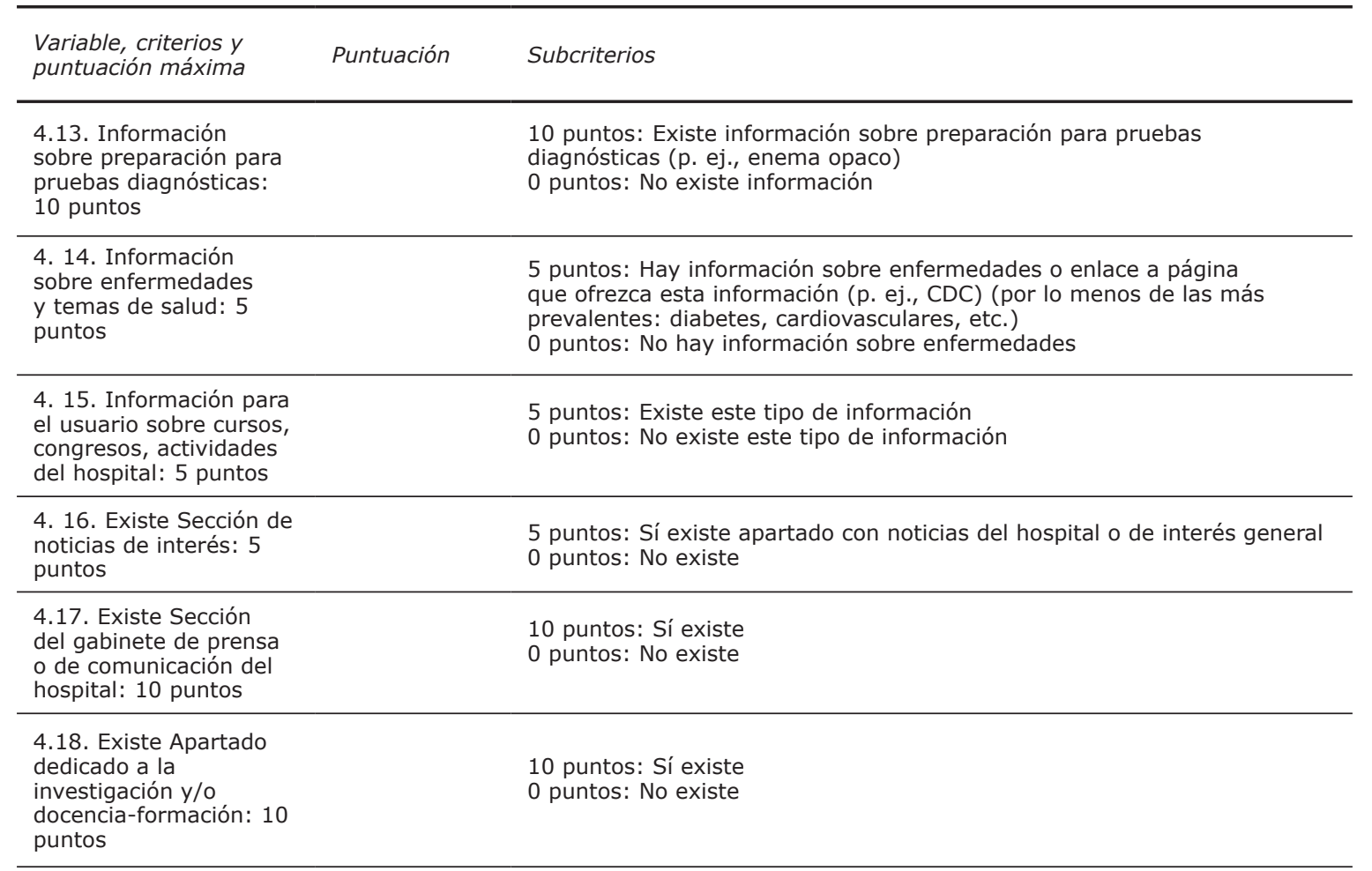

\section{Actualización de \\ los contenidos \\ 10 puntos}

\begin{tabular}{ll}
\hline 5.1. Existencia de fecha & 5 puntos: Sí \\
$\begin{array}{l}\text { de actualización: } 5 \\
\text { puntos }\end{array}$ & 0 puntos: No \\
\hline
\end{tabular}

5.2. Actualización de la 5 puntos: Información actualizada (menos de 1 mes entre la fecha de información: 5 puntos revisión de la web y la fecha de la información)

3 puntos: Información reciente (entre 1 y 6 meses)

0 puntos: Más de 6 meses no actualizada

\section{Referentes de}

calidad

10 puntos

6.1. La web cumple algún criterio de calidad específico para webs de salud (Hon Code, WMA, ACSA): 10 puntos
10 puntos: Aparece al menos un sello acreditador de calidad de la web 0 puntos: No se ha adherido a ningún sello de calidad

\section{Información para \\ el profesional \\ 7.1. Existe apartado para el profesional: 20 puntos}

20 puntos: Sí hay apartado específico para los profesionales del centro (acceso a intranet o similar)

0 puntos: No hay

\section{Información para \\ proveedores \\ 8.1. Existe apartado \\ para proveedores: \\ 10 puntos}

10 puntos: Sí hay apartado específico para los proveedores del centro

0 puntos: No hay 


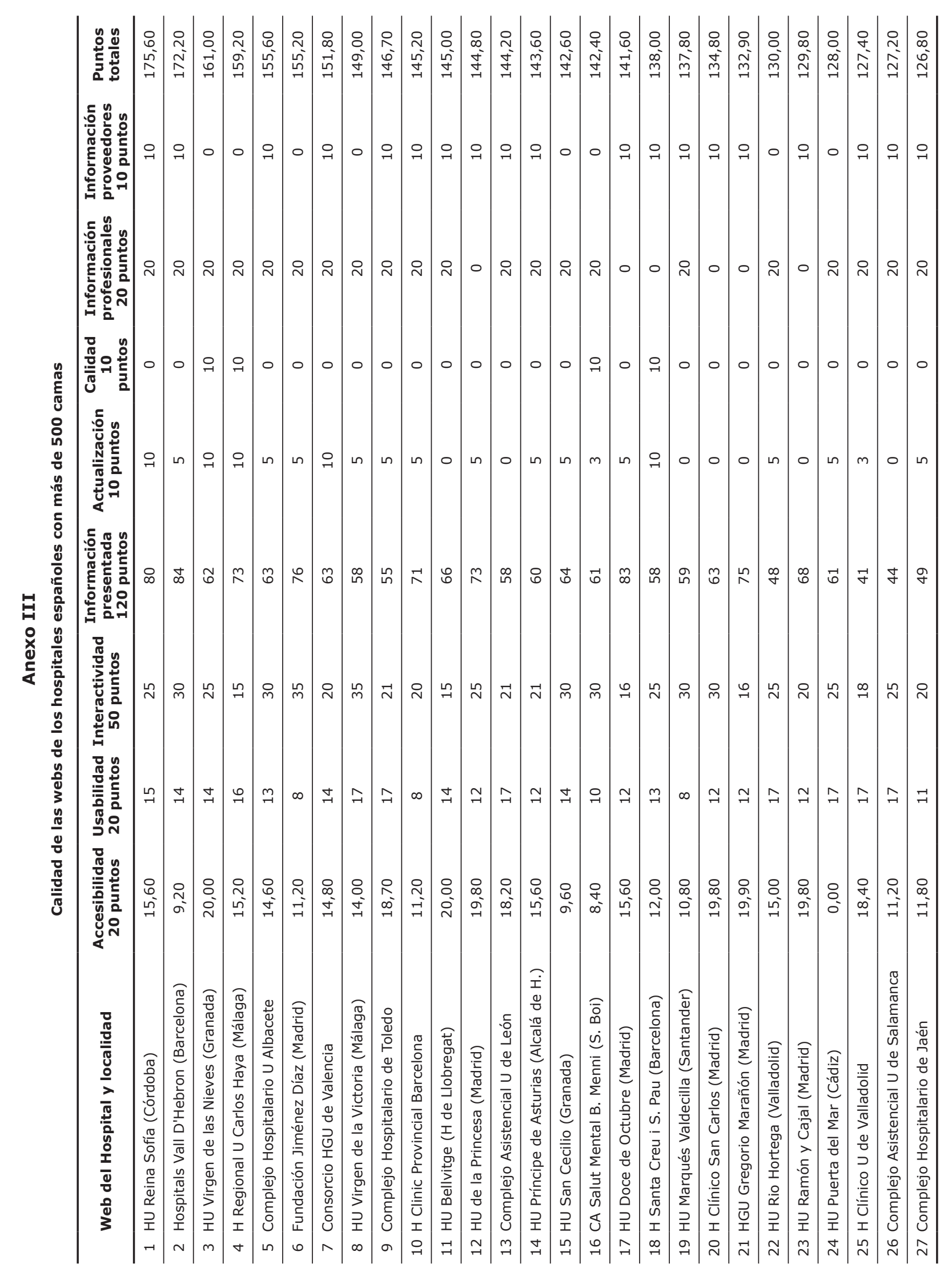




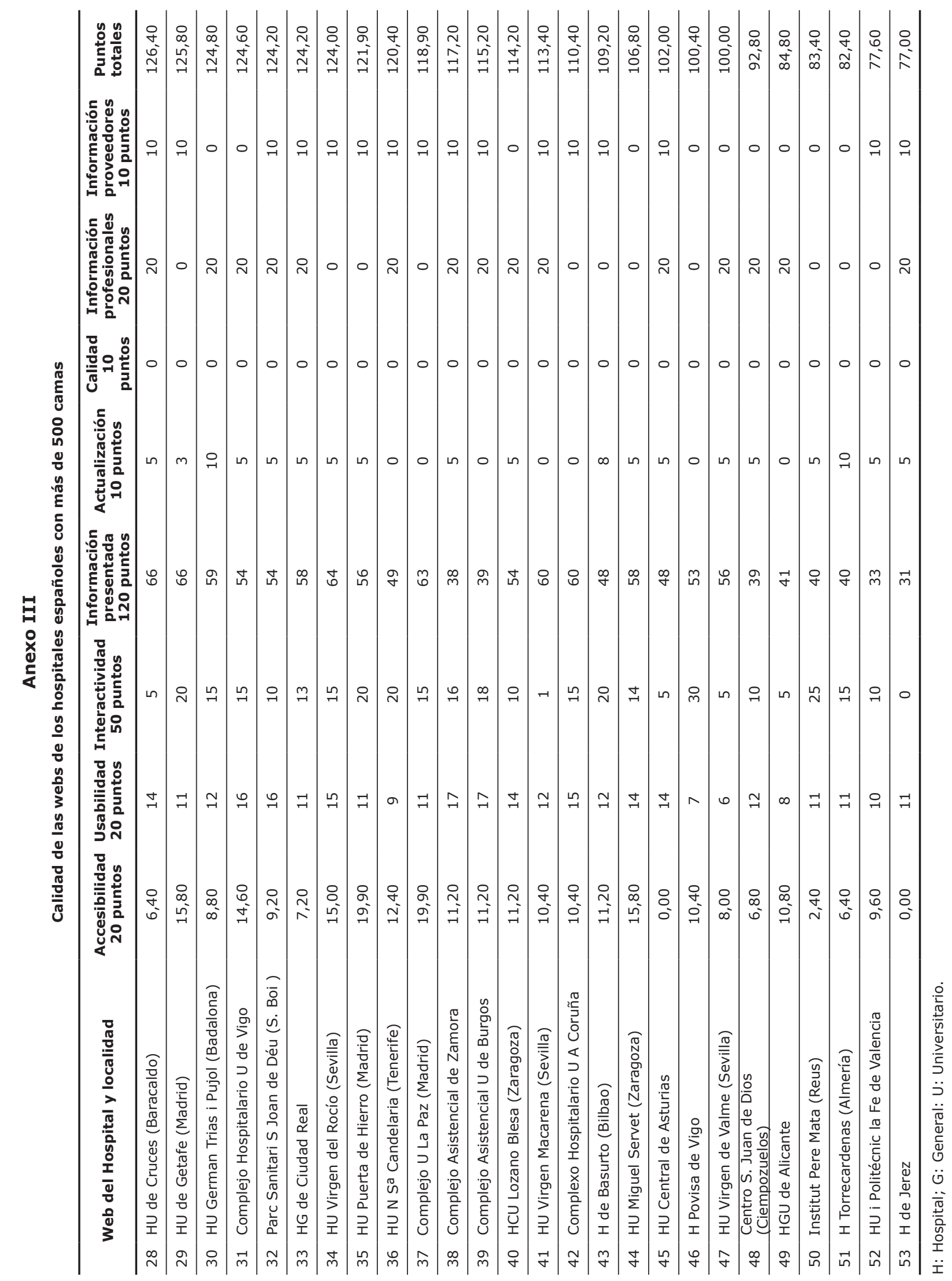

